\title{
A brief introduction to multivariate methods in grape and wine analysis
}

\author{
D Cozzolino' \\ W U Cynkar' \\ N Shah' \\ R G Dambergs ${ }^{2}$ \\ P A Smith'
}

'The Australian Wine Research Institute, Urrbrae, Glen Osmond, SA, Australia; ${ }^{2}$ The Australian Wine Research Institute, Tasmanian Institute of Agricultural Research, University of Tasmania, Hobart, Tasmania, Australia

Correspondence: Daniel Cozzolino The Australian Wine Research Institute. Waite Road, Urrbrae. PO Box 197, Glen Osmond SA 5064, Australia Fax:+6I 88303660 I

Email daniel.cozzolino@awri.com.au

\begin{abstract}
Real-world systems are usually multivariate and hence usually cannot be adequately described by one selected variable without the risk of serious misrepresentation. Analyzing the effect of one variable at a time by analysis of variance techniques can give useful descriptive information, but this will not give specific information about relationships among variables and other important relationships in the entire matrix. Multivariate data analysis was developed in the late 1960s, and used by a number of research groups in analytical and physical organic chemistry due to the introduction of instrumentation giving multivariate responses for each sample analyzed. Development of such methods was also made possible by the availability of computers. Multivariate data analysis involves the use of mathematical and statistical techniques to extract information from complex data sets. The objective of this paper is to briefly describe and illustrate some multivariate data analysis methods used for grape and wine analysis.
\end{abstract}

Keywords: multivariate analysis, data mining, wine, grape

\section{Introduction}

Scientists in the food and beverage industry are faced with many different quality control tasks, including verifying that products meet required compositional and flavor standards, identifying changes in process parameters that might lead to a change in quality, detecting adulteration in raw materials and manufactured products, and identifying geographical origin of raw materials. ${ }^{1-3}$

Traditionally, much of the research in the field of grape and wine has been conducted in a manner that can be described as "univariate", since it only examined the effect (response) of a single variable on the overall matrix. ${ }^{4-5}$ Around the 1920 s, when many statistical methods were developed, samples were considered cheap and measurements expensive. ${ }^{6}$ Since that time, the nature of technology has changed, samples are now expensive (eg, the high cost of experiments) and measurements are cheap.

Analyzing the effect of one variable at a time by analysis of variance (ANOVA) techniques can give useful descriptive information, but this will not give specific information about relationships among variables and other important relationships in the entire matrix..$^{7-11}$ Multivariate data analysis was developed in the late 1960s and was used by a number of research groups in analytical and physical organic chemistry. Its development was due to the introduction of modern instrumentation, which gave multivariate responses for each sample analyzed (eg, wavelengths, ions, mass to charge ratios, chromatographic peaks) and to the wider availability of computers. ${ }^{9-13}$

In modern chemical measurements, we are often confronted with so much data that the essential information may be not readily evident. ${ }^{9-13}$ Certainly that can be the case with chromatographic or spectral data for which many different observations (peaks or wavelengths) have been collected from a single sample. Each different measurement can be thought of as a different dimension. Traditionally, analysts strive to eliminate matrix interference in analytical methods by isolating or extracting the analyte in 
order to make the measurement itself apparently simple and certain. However, this approach ignores the possible effects of chemical and physical interactions between the different constituents present in the sample, which is something that is especially evident when complex materials like grapes and wine are analysed. ${ }^{14-19}$

Univariate models do not consider the contributions of more than one variable source and can result in models that oversimplify the system under analysis. Therefore, in the modern scientific approach, scientists need to look at the sample in its entirety and not just at a single component if we wish to untangle all the complicated interactions between the constituents and understand their combined effects on the whole matrix. ${ }^{20}$ Multivariate methods provide the means to move beyond the one-dimensional (univariate) world. In many cases, multivariate analysis can reveal constituents that are important through various interferences and interactions. This inductive rather than deductive reasoning needs a leap of imagination; it might be harder to accept and seemingly less certain and secure, but it opens a new chapter in the development of inferences from the analysis of the whole matrix..$^{9-13,20}$ Therefore, multivariate data analysis is defined as the application of mathematical or statistical methods to chemical data.

Multivariate data analysis, unlike classic statistics, considers multiple variables simultaneously. Although multivariate ANOVA (MANOVA) is available, other multivariate analytical methods have the advantage that they can take collinearity into account. ${ }^{7-8}$ Multivariate analysis can take into account the variation in one variable, or a group of variables, in terms of co-variation with other variables. ${ }^{8,20-22}$ The analysis can mathematically describe the co-variation (degree of association) between variables, or find a mathematical function (regression model), by which the values of the dependent variables are calculated from values of the measured (independent) variables. ${ }^{20-22}$

Today, many food quality measurement techniques are multivariate and based on indirect measurements of chemical, physical, and sensory properties. ${ }^{5-8}$ A typical characteristic of many of the most useful of these instrumental techniques is that, paradoxically, the measurement variable might not have a direct relationship with the property of interest, for instance the concentration of a particular chemical in the sample, that is, a correlative method. This often refers to chemical and physical interference. ${ }^{20-22}$

Multivariate data analysis research spans a wide area of different methods which can be applied in different fields of science and technology. They include methods and techniques for collecting good data (optimization of experimental parameters, design of experiments, calibration, signal processing) and for getting information from these data (statistics, pattern recognition, modeling, structureproperty-relationship estimations). ${ }^{8,11}$ The most commonly used multivariate data analysis techniques applied to grape and wine analysis are principal component analysis (PCA) and partial least squares (PLS) regression. ${ }^{9-13}$

The aim of this review is to give a brief description of different multivariate methods used in grape and wine analysis.

\section{Brief description of multivariate data methods}

Both quantitative and qualitative applications of multivariate data analysis have been reported in the literature for the analysis of grape and wine. Most involve the use of PCA and PLS regression. ${ }^{14-19,23-43}$ The following section describes briefly the different multivariate data analysis methods used. Many of the methods are based on the concept of soft modeling, a linear modeling method that originated in the field of multivariate statistical analysis, but which has become synonymous with the term "chemometrics". The focus of the soft modeling method on the properties of the signal, rather than on the noise, helps to distinguish multivariate data analysis from statistics, where the emphasis is usually on the structure and properties of the error term. ${ }^{9-13}$ Chemists often confuse the two fields, but remembering the difference in focus makes distinguishing them relatively simple. ${ }^{9-13}$

Because of the heavy emphasis on soft modeling in multivariate data analysis, the field developed around an algorithmic rather than theoretical framework, an attribute that is now beginning to change. ${ }^{4,12-13,20-22}$ Discussions of soft modeling in current literature are more likely to focus on the linear algebraic theory of the modeling than on the specific steps needed to form the model. ${ }^{4,12-13,20-22}$ It is useful to have an appreciation for some of the key approaches and assumptions of soft modeling, as these underlie the logic of many of the multivariate analytical methods. ${ }^{10-13}$ For example modeling in spectroscopy has been done using "first-principles" (hard) models. A hard model is one that describes the system in terms of a mathematical relationship developed using the measurement variables as independent variables and the desired outputs as dependent variables..$^{7-8}$ Because chemical systems are generally complex, the hard modeling used in chemistry has either been applied to simplified systems or has involved either limiting "laws" or other approximations and restrictions to the region of application 
of the hard model..$^{7-8}$ The ubiquitous “Beer's Law" relations used in quantitative spectroscopy is an example of one such first-principles model with well known limitations due to the simplifications made in the theory. ${ }^{20-22}$

Soft modeling sees the modeling problem from an entirely different logical perspective. It assumes that the chemical system under study is complex and that it is not possible or economically feasible to adequately describe the behavior of the system using a hard model. The soft model is based on variation and correlation in the data, as captured in a covariance matrix, which can be thought of as a measure of the overall fluctuation in each independent variable present in the data set, as well as the variable-variable interactions. ${ }^{20-22}$

The first step in soft modeling is to express the data in terms of a new set of axes based on the different contributions to variation in the data. ${ }^{7-8,20-22}$ This is conveniently done by a mathematical conversion from measured data to new axes based on covariance in the data set. A set of orthogonal components made from linear combinations of the independent (eg, spectral data) variables is created to describe independent sources of the observed variation in the covariance matrix created from the data set analyzed, according to the equation:

$$
\mathrm{X}=\mathrm{U}^{\mathrm{VT}}
$$

where matrix $\mathrm{V}$, the loadings of the set of spectral data described by matrix $\mathrm{X}$, contains the linear combinations of the original measurement variables that define the new variationbased coordinate system spanning the data in $\mathrm{X}$ and matrix $\mathrm{U}$, called the scores of $X$, contains the coordinates of the data $X$ in that variation-based coordinate system..$^{20-22}$

The linear combinations of the measured spectral variables that result from the rotation are called latent variables because they are derived rather than measured. The latent variables extracted to describe a data set are ordered in terms of the size of the independent sources of variation that they explain. The first latent variable explains the largest independent source of variance in the data, the second latent variable the second largest, and so on, until all variation in the data set is accounted for by one of the linear combinations of the measurements. ${ }^{8,20-22}$

The second step in soft modeling is the elimination of noninformative latent variables. Generally, re-expression of the data set $\mathrm{X}$ in terms of latent variables is not useful unless a decision is also made on the number of latent variables that are needed to adequately explain the systematic variation in the data X. At this point it is important to distinguish between the variables that help answer the questions posed (and therefore must contain "signal") and those that do not help (because they contain "noise"). , $20-22^{2}$

\section{Exploratory data analysis}

Patterns of association exist in many data sets, but the relationships between samples can be difficult to discover when the data matrix exceeds three or more variables. ${ }^{20-22}$ Exploratory data analysis can reveal hidden patterns in complex data by reducing the information to a more comprehensible form. Such multivariate data analysis can expose possible outliers and indicate whether there are patterns or trends in the data. Exploratory algorithms such as PCA and hierarchical cluster analysis (HCA) are designed to reduce large complex data sets into a series of optimized and interpretable views. ${ }^{8,13}$

\section{Principal component analysis}

PCA is used as a tool for screening, extracting, and compressing multivariate data. ${ }^{8,10-13}$ PCA employs a mathematical procedure that transforms a set of possibly correlated response variables into a new set of noncorrelated variables called principal components. ${ }^{10-13}$ PCA can be performed on either a data matrix or a correlation matrix depending on the type of variables being measured. However, in a case where the original variables are nearly noncorrelated, nothing can be gained by using a PCA analysis. PCA produces linear combinations of variables that are useful descriptors or even predictors of some particular structure in the data matrix. ${ }^{8,11-13}$

PCA is one of the most commonly used methods to analyze large data sets and has been applied to different types of instrumental methods as well as sensory data. For example, recent research has shown that rapid analysis of wine volatiles using an electronic nose (EN) instrument produces signals containing information that, even without chromatographic separation, can be used to determine a fingerprint of wine based on its aroma profile. ${ }^{23-29}$ The combination of PCA analysis and gas sensors allow the detection of mass fragments formed during ionization of volatile compounds. ${ }^{23-29}$ This suggests that some of these volatiles are directly responsible for the sensory differences between samples and measuring the mass fragments of these compounds can provide some understanding of the chemical basis for sensory differentiation as well as a basis for varietal discrimination. ${ }^{23-29}$ Other examples of the use of PCA applied to chemical, instrumental, and sensory data are shown in Table 1.

A combination of PCA and rapid methods is illustrated in Figure 1. The mid infrared (MIR) spectra (Figure 1A) of a set of wine samples (Shiraz, Riesling, and Sauvignon Blanc) 
Table I Use of principal component analysis and discriminant analysis combined with instrumental methods in the grape and wine industry

\begin{tabular}{lll}
\hline Reference & Instrumental method & Sample \\
\hline 16 & NIR & $\begin{array}{l}\text { Discrimination of Riesling } \\
\text { and Chardonnay wines }\end{array}$ \\
29 & Electronic nose & Wine spoilage \\
30 & Electronic nose & Wine classification \\
31 & Electronic nose & Wine classification \\
35 & and tongue & \\
36 & Electronic nose & Aroma compounds \\
47 & NIR & Fermentation monitoring \\
\hline
\end{tabular}

Abbreviations: NIR, near infrared spectroscopy; MIR, mid infrared spectroscopy.

and their principal component scores (Figure 1B) are plotted, indicating a separation between the wine samples analyzed. From the visual observation of the MIR spectra alone it is very hard to identify groups of samples related to variety. The use of PCA as a multivariate analytical tool allows the classification of wine samples according to variety (Shiraz, Riesling, and Sauvignon Blanc).

\section{Discriminant analysis using PLS regression}

Discriminant analysis (DA) can be considered a qualitative calibration method. Instead of calibrating for a continuous variable, one calibrates for group membership (categories). In the case of two groups (eg, A and B) and several measurements there is a fairly obvious way to use regression methods to perform a DA. For example, create an artificial (dummy) Y-variable that has value 0 (zero) for each case in group $\mathrm{A}$, and 1 (one) for each in group B, and develop a regression equation between $\mathrm{Y}$ and the $\mathrm{X}$-variable measurements. The resulting models are evaluated in terms of their predictive ability to predict the $\mathrm{Y}$ variable of new and unknown samples (standard error of prediction, SEP). The use of DA and other classification tools can be found extensively in the analysis of grape and wine. ${ }^{14,29-31}$

Several studies have suggested that gas sensors together with DA methods might be used by the wine industry for the identification of white wine varieties or their blends. ${ }^{27-29}$ Even though the conventional analysis based on gas chromatography provides fundamental information about the volatile compounds present in the wine, the use of gas sensors has advantages of simplicity of sample preparation and reduced time of analysis. ${ }^{27-29}$ For example, the use of EN was reported to classify Riesling and unwooded Chardonnay wines from Australia and to discriminate between different wines, regions, vintages, and spoilage. ${ }^{29-32}$

In another study, metal oxide sensor (MOS) instruments were tested for the ability to characterize Ontario-produced fruit wines. ${ }^{33}$ Eight fruit wines (blueberry, cherry, raspberry, blackcurrant, elderberry, cranberry, apple, and peach) and four grape wines (red, Chardonnay, Riesling, and ice wines) were each obtained from five Ontario wineries. This study showed that it was possible to separate each wine variety based on differences between wineries. ${ }^{33}$ The results show that MOS instruments can discriminate between fruit and grape wines and may become an important tool for standardization of wine quality. ${ }^{33}$
A

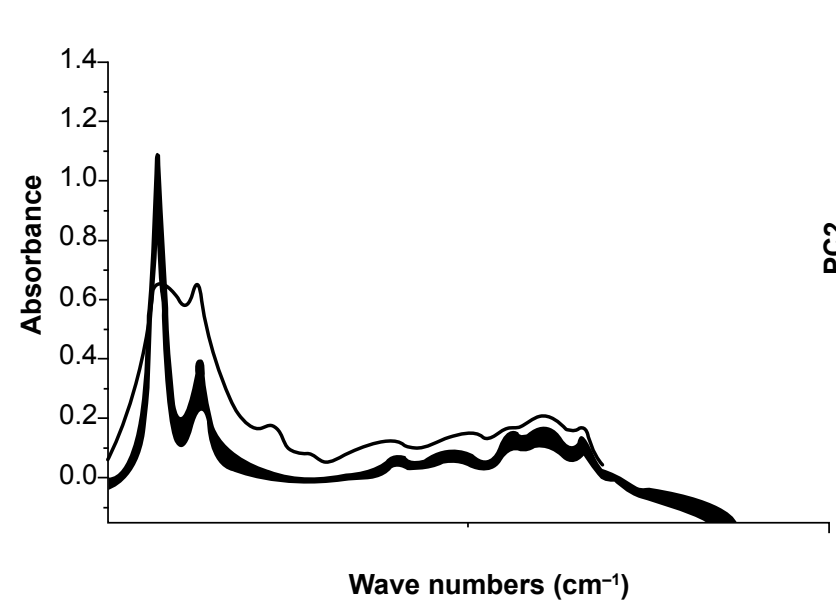

B

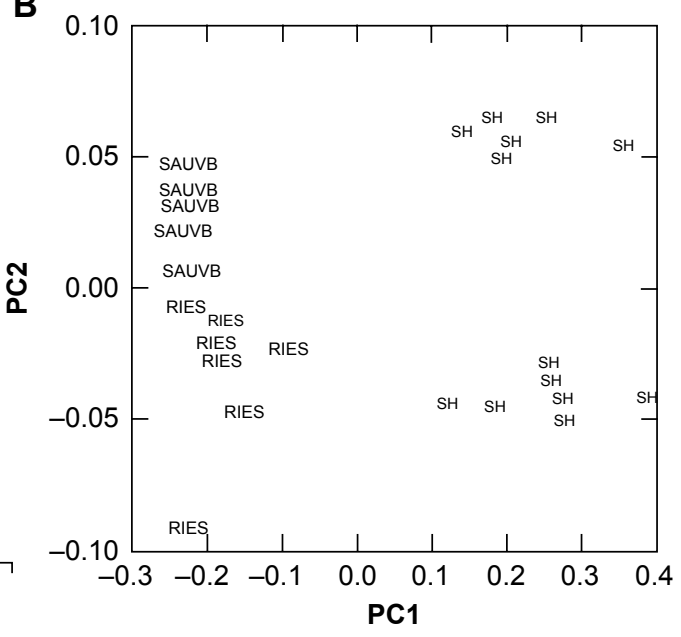

Figure I A combination of PCA and rapid methods. A the mid-infrared spectra of a set of wine samples, $\mathbf{B}$ and their principal component scores. Abbreviations: PCA, principal component analysis; PC, principal component score. 
An application of EN (based on a tin oxide array) for the identification of typical aromatic compounds present in white and red wines and responsible for different aroma attributes (eg, fruity, floral, herbaceous, vegetative, spicy, smoky) has also been reported. ${ }^{34,35}$ Both PCA and DA showed that datasets of these groups of compounds were clearly separated and confirmed that the system could correctly discriminate the aromatic compounds added to the wine..$^{34,35}$

\section{Classification and clustering}

Many applications require that samples be assigned to predefined categories, or "classes". This may involve determining whether a sample is good or bad, or predicting an unknown sample as belonging to one of several distinct groups. A classification model is used to predict a sample class by comparing the sample to a previously analyzed experience set, in which categories are already known. Both k-nearest neighbour (KNN) and soft independent modeling of class analogy (SIMCA) are primary multivariate data analysis workhorses. ${ }^{11-13}$ When these techniques are combined to create a classification model, the answers provided are more reliable and include the ability to reveal unusual samples or patterns in the data. ${ }^{11-13}$

\section{Quantitative analysis: modeling and calibration}

In many applications, it is expensive, time consuming or difficult to measure a property of interest directly. Such cases require the analyst to predict the property of interest based on related properties that are easier to measure. The goal of multivariate data analysis regression analysis is to develop a calibration model which correlates the information in the set of known measurements to the desired property. Multivariate data analysis algorithms for performing regression include PLS and principal component regression (PCR) and are designed to avoid problems associated with noise and correlations in the data. Because the regression algorithms used are based on factor analysis, the entire group of known measurements is considered simultaneously, and information about correlations among the variables is automatically built into the calibration model. Multivariate data analysis regression lends itself handily to the on-line monitoring and process control industry, where fast and inexpensive systems are needed to test, predict, and make decisions about product quality. ${ }^{36}$

The modeling (regression) of one or several dependent $\mathrm{Y}$-variables by means of a set of predictor responses (X-variables), is one of the most common applications of multivariate data analysis in science and technology. This is known as calibration and is one of the most important tasks in quantitative analysis. ${ }^{4,8,9}$ The principal aim is to undertake regression analysis to develop a suitable mathematical model for descriptive or predictive purposes. ${ }^{12}$ Examples of mathematical models that help to deal with highly correlated data are PCR and PLS regression. ${ }^{4,89}$

\section{Partial least squares regression}

PLS regression is a recently developed generalization of multiple linear regression (MLR)., ${ }^{4,9}$ PLS regression is of particular interest because, unlike MLR, it can analyse data with strongly collinear (correlated), noisy and redundant variables (X variables) and can also model several characteristics (Y values) at the same time. ${ }^{4,8,9}$ Note that the emphasis is on predicting the characteristics and not necessarily on trying to understand the underlying relationships between the variables. PLS is a data reduction technique in that it reduces the $\mathrm{X}$-variables to a set of noncorrelated factors that describe the variation in the data. However, if the number of factors used in the regression model is too large (greater than the number of samples) the models that fit the sampled data perfectly might fail to predict new data. ${ }^{4,89}$ This phenomenon is called overfitting and is discussed further below; as the number of factors is increased with a PLS calibration it approaches the model of multiple linear regression, which is notoriously prone to overfitting when there is a large amount of $\mathrm{X}$ data relative to $\mathrm{Y}$ data to be predicted. ${ }^{4,8,9}$

A variant of PLS regression is locally weighted regression (LWR). ${ }^{37-40}$ A potential problem with using the whole data matrix is that large sample matrix variations within the dataset can create problems in developing a global PLS calibration, capable of predicting all samples. ${ }^{37-40}$ This can be a particular problem with agricultural products such as grape samples that can show seasonal variations and variations due to environmental effects. For example it has been demonstrated that seasonal and varietal variations can place constraints on calibration performance in the analysis of grapes with PLS regression of near infrared (NIR) spectra. When attempting to produce a universal calibration spanning many seasons, growing regions and grape varieties, the performance of PLS regression in prediction of grape anthocyanin was limited by pronounced nonlinearity. ${ }^{37-40}$ A potential method to minimize this matrix-related error is the use of "locally weighted" a regression algorithm that develops a PLS calibration from samples that best match the sample to be predicted. A particular software variant of LWR is the LOCAL algorithm, that uses a software driven selection process to match unknown samples with samples from a large calibration database, then uses a PLS calibration tailored to fit the unknown sample. It has been shown that 
LOCAL improves calibration performance over standard PLS, allowing universal application of calibrations for grape analytes across the wine industry, regardless of growing region, grape variety, and growing season. ${ }^{37-40}$

Table 2 summarizes some applications of PLS as a regression method to predict several compounds in grape, juice, and wine samples.

\section{Artificial neural networks}

Application of artificial neural networks (ANN) is a more recent technique for data and knowledge processing, characterized by its analogy with a biological neuron. ${ }^{10}$ When the firing frequency of a neuron is compared with that of a computer, then for a neuron this frequency is rather low. ${ }^{10,51}$ In the biological neuron the input signal from the dendrites travels through the axons to the synapse. There the information is transformed and sent across the synapse to the dendrites of the next neuron forming part of a highly complex network. The multivariate techniques based on ANN simulates the biological neuron by multiplication of the input signal $(\mathrm{X})$ with the synaptic weight $(\mathrm{W})$ to derive the output signal (Y). ${ }^{10,51}$ Unlike linear regression, PCR and PLS, ANN can deal with nonlinear relationships between variables. The performance of ANN is attributed to a high degree of interconnections. Like LOCAL regression, ANN can handle nonlinearity and outperforms standard PLS regression in that situation. ${ }^{34-36}$

\section{Overfitting and underfitting}

When using any of the data modeling techniques presented above, it is important to select an optimum number of variables or components. ${ }^{8}$ If too many are used, too much

Table 2 Use of partial least squares regression combined with instrumental methods to measure chemical compounds in grape and wine samples

\begin{tabular}{|c|c|c|c|c|}
\hline Reference & Parameter & Sample & $\begin{array}{l}\text { Instrumental } \\
\text { method }\end{array}$ & SEP \\
\hline \multirow[t]{5}{*}{40} & Alcoholic degree $(\%, v / v)$ & Red, rose and white wines & $\mathrm{VIS}+\mathrm{NIR}$ & 0.24 \\
\hline & Total acidity (meq $\mathrm{L}^{-1}$ ) & & & 0.48 \\
\hline & $\mathrm{pH}$ & & & 0.07 \\
\hline & Glycerol ( $\left.\mathrm{g} \mathrm{L}^{-1}\right)$ & & & 0.72 \\
\hline & Reducing sugars $\left(\mathrm{g} \mathrm{L}^{-1}\right)$ & & & 0.33 \\
\hline \multirow[t]{4}{*}{$4 I$} & Sodium $\left(m g L^{-1}\right)$ & White wine & VIS + NIR & 9.22 \\
\hline & Potassium (mg L-1) & & & 79.0 \\
\hline & Magnesium (mg L-1) & & & 14.5 \\
\hline & Calcium (mg L-1) & & & 8.09 \\
\hline \multirow[t]{3}{*}{42} & Sugar (Brix) & White varieties & NIR & 0.31 \\
\hline & Malic acid $\left(\mathrm{g} \mathrm{L}^{-1}\right)$ & (must and wine) & & 1.02 \\
\hline & Lactic acid $\left(\mathrm{g} \mathrm{L}^{-1}\right)$ & & & 1.34 \\
\hline 18 & Methanol $\left(g \mathrm{~L}^{-1}\right)$ & Grape spirit & NIR & 0.06 \\
\hline \multirow[t]{7}{*}{44} & Alcohol (vol \%) & $\begin{array}{l}\text { Several varieties } \\
\text { and styles }\end{array}$ & MIR & 0.02 \\
\hline & Alcohol $\left(\mathrm{g} \mathrm{L}^{-1}\right)$ & & & 1.96 \\
\hline & Volatile acidity $\left(\mathrm{g} \mathrm{L}^{-1}\right)$ & & & 0.008 \\
\hline & $\mathrm{pH}$ & & & 0.01 \\
\hline & Total acid $\left(\mathrm{g} \mathrm{L}^{-1}\right)$ & & & 0.06 \\
\hline & Fructose $\left(\mathrm{g} \mathrm{L}^{-1}\right)$ & & & 1.21 \\
\hline & Glucose $\left(\mathrm{g} \mathrm{L}^{-1}\right)$ & & & 0.49 \\
\hline \multirow[t]{2}{*}{43} & Malvidin-3-glucoside $\left(\mathrm{mg} \mathrm{L}^{-1}\right)$ & $\begin{array}{l}\text { Several varieties } \\
\text { and styles }\end{array}$ & Spectroscopy & 0.02 \\
\hline & Total anthocyanins (mg L-1) & & & 1.96 \\
\hline 45 & Polysaccharides & White wine & MIR & \\
\hline
\end{tabular}

Abbreviations: VIS + NIR, visible plus near infrared spectroscopy; MIR, mid infrared spectroscopy; SEP, standard error of prediction. 
redundancy in the $\mathrm{X}$-variables is modeled and the solution can become overfitted; the model will be very dependent on the dataset and will give poor prediction results. On the other hand, using too few components will cause underfitting and the model will not be large enough to capture the variability in the data. This "fitting" effect is strongly dependent on the number of samples used to develop the model, and in general, more samples give rise to more accurate predictions. ${ }^{8}$

\section{Conclusions}

Multivariate data analytical methods both quantitative and qualitative are increasingly being used by research scientists in combination with traditional analytical tools. Multivariate data analysis techniques can be used to simplify methods and reduce analytical times for many compounds present in grapes and wine. Key factors contributing to the use of these methods in the grape and wine sector have been advances in instrument reliability, readily available multivariate data analysis software, and improved computing power. Compared to traditional methods, multivariate analysis combined with modern instrumental techniques (eg, EN, high-performance liquid chromatography, NIR and MIR spectrophotometers) often give new and better insight into complex problems by measuring a greater number of chemical compounds at once, thus enabling the "fingerprinting" of each sample. These methods are attractive due to their inherent features of versatility, flexibility, effectiveness, and richness of information.

\section{Acknowledgments}

Comments and suggestions made by reviewers and editor in the original manuscript are acknowledged. This project is supported by Australia's grapegrowers and winemakers through their investment body the Grape and Wine Research and Development Corporation, with matching funds from the Australian government. The work was conducted by The Australian Wine Research Institute.

\section{References}

1. Downey G. Authentication of food and food ingredients by near infrared spectroscopy. J Near Infrared Spec. 1996;4:47-61.

2. Downey G. Food and food ingredient authentication by mid-infrared spectroscopy and chemometrics. Trends Anal Chem. 1998;17: $418-424$.

3. Arhurst PR, Dennis MJ. Food Authentication. London, UK: Blackie Academic and Professional; 1996.

4. Munck L, Norgaard L, Engelsen SB, Bro R, Andersson CA. Chemometrics in food science: a demonstration of the feasibility of a highly exploratory, inductive evaluation strategy of fundamental scientific significance. Chem Intell Lab Sys. 1998;44:31-60.

5. Francis IL, Høj PB, Dambergs RG, et al. In: Blair R, Williams P, Pretorious IS, editors. Proceedings 12th Australian Wine Industry Technical Conference. Adelaide, Australia: Winetitles; 2005.
6. Bendell A, Disney J, McCollin C. The future role of statistics in quality engineering and management. The Statistician. 1999;48:299-326.

7. Martens H, Martens M. Multivariate Analysis of Quality. An Introduction. Hoboken, NJ: John Wiley and Sons, Ltd; 2000.

8. Naes T, Isaksson T, Fearn T, Davies T. A User-friendly guide to multivariate calibration and classification. Chichester, UK: NIR Publications; 2002.

9. Wold S, Sjöstrom M, Eriksson L. PLS-regression: a basic tool of chemometrics. Chem Intell Lab Sys. 2001;58:109-130.

10. Otto M. Chemometrics: Statistics and computer application in analytical chemistry. Hoboken, NJ; Wiley-VCH; 1999.

11. Siebert KJ. Chemometrics in brewing: a review. J Amer Soc Brew Chem. 2001;59:147-156.

12. Adams MJ. Chemometrics in analytical spectroscopy (RSC Spectroscopy Monographs). London, UK: The Royal Society of Chemistry; 1995.

13. Brereton RG. Introduction to multivariate calibration in analytical chemistry. The Analyst. 2000;125:2125-2154.

14. Arvantoyannis I, Katsota MN, Psarra P, Soufleros E, Kallinthraka S. Application of quality control methods for assessing wine authenticity: Use of multivariate analysis (chemometrics). Trends Food Sci Technol. 1999;10:321-336.

15. Cordella Ch, Moussa I, Martel A-C, Sbirrazzuoli N, Lizzani-Cuvelier L. Recent developments in food characterisation and adulteration detection: technique-oriented perspectives. J Agric Food Chem. 2000;50: 1751-1764.

16. Cozzolino D, Smyth HE, Gishen M. Feasibility study on the use of visible and near infrared spectroscopy to discriminate between white wine of different varietal origin. J Agric Food Chem. 2003;54: 7703-7708.

17. Cozzolino D, Kwiatkowski MY, Parker M, et al. Prediction of phenolic compounds in red wine fermentations by visible and near infrared spectroscopy. Anal Chim Acta. 2003;513:73-80.

18. Dambergs RG, Kambouris A, Francis IL, Gishen M. Rapid analysis of methanol in grape-derived distillation products using near infrared transmission spectroscopy. J Agric Food Chem. 2002;50:3079-3084.

19. Dambergs RG, Esler MB, Gishen M. Application in analysis of beverages and brewing products. In: Roberts CA, Workman J, Reeves JB, editors. Near Infrared Spectroscopy in Agriculture. Madison: Agronomy Monograph; 2004.

20. Geladi P. Chemometrics in spectroscopy. Part I. Classical chemometrics. Spectrochimica Acta Part B. 2003;58:767-782.

21. Wold S. Chemometrics; what do we mean with it, and what do we want from it? Chem Intell Lab Sys. 1995;30:109-115.

22. Wise BM, Gallagher NB. The process chemometrics approach to process monitoring and fault detection. J Proc Cont. 1996;6:329-348.

23. Di Natale C, Davide FAM, D'Amico A, et al. Complex chemical pattern recognition with sensor array: the discrimination of vintage years of wine. Sensor Actuat. 1995;25:801-804.

24. Di Natale C, Paolesse R, Burgio M, Martinelli E, Pennazza G, D’Amico A. Application of metalloporphyrins-based gas and liquid sensor arrays to the analysis of red wine. Anal Chim Acta. 2004;513:49-56.

25. Di Natale C, Paolesse R, Macagnano A, et al. Application of a combined artificial olfaction and taste system to the quantification of relevant compounds in red wine. Sensor Actuat. 2000;69:342-347

26. Gutierrez A, Burgos JA, Garcera C, Padilla AI, Zarzo M, Chirivella C, Ruiz M L, Molto E. Optimization of an aroma sensor for assessing grape quality for wine making. Spanish J Agric Res. 2007;5:157-163.

27. Deisingh AK, Stone DC, Thompson M. Applications of electronic noses and tongues in food analysis. Int J Food Sci Tech. 2004;39:587-604.

28. Cozzolino D, Smyth HE, Lattey KA, et al. Combining mass spectrometry based electronic nose, visible-near infrared spectroscopy and chemometrics to assess the sensory properties of Australian Riesling wines. Anal Chim Acta. 2006;563:319-324.

29. Cynkar WU, Cozzolino D, Dambergs RG, Janik L, Gishen M. Feasibility study on the use of a head space mass spectrometry electronic nose (MS e-nose) to monitor red wine spoilage induced by Brettanomyces yeast. Sensor Actuat. 2007;124:167-171. 
30. Aleixandre M, Lozano J, Gutierrez J, Sayago I, Fernandez MJ, Horrillo MC. Portable e-nose to classify different kinds of wine. Sensors and Actuators. 2008;131:71-76.

31. Buratti S, Bendetti S, Scampicchio M, Pangerod EC. Characterization and classification of Italian Barbera wines by using an electronic nose and an amperometric electronic tongue. Anal Chim Acta. 2004;525: 133-139.

32. Penza M, Cassano G. Chemometric characterization of Italian wines by thin-film multisensors array and artificial neural networks. Food Chem. 2004;86:283-296.

33. McKellar RC, Vasantha-Rupasinghe HP, Xuewen L, Knight KP. The electronic nose as a tool for the classification of fruit and grape wines from different Ontario wineries. J Sci Food Agric. 2005;85:2391-2396.

34. Lozano J, Santos JP, Aleixandre M, Sayago I, Gutierrez J, Horrillo MC. Identification of typical wine aromas by means of electronic nose. IEEE Sensors J. 2006;6:173-178.

35. Lozano J, Santos JP, Horrillo MC. Classification of white wine aromas with an electronic nose. Talanta. 2005;67:610-617.

36. Cozzolino D, Parker M, Dambergs RG, Herderich M, Gishen M. Chemometrics and visible-near infrared spectroscopic monitoring of red wine fermentation in a pilot scale. Biotech Bioeng. 2006;95: 1101-1107.

37. Esler MB, Gishen M, Francis IL, et al. Effects of variety and region on new infrared reflectance spectroscopic analysis of quality parameters in red wine grapes. In: Cho RK, Davies AMC, editors. Proceedings of the 10th International Conference. Chichester, UK: NIR Publications; 2002.

38. Shenk JS, Berzaghi P, Westerhaus MO. Investigation of a LOCAL calibration procedure for near infrared instruments. $J$ Near Infrared Spec. 1997;5:223-226.

39. Dambergs RG, Cozzolino D, Cynkar WU, Janik L, Gishen M. The determination of red-grape quality parameters using the LOCAL algorithm. J Near Infrared Spec. 2006;14:71-79.

40. Urbano-Cuadrado M, Luque de Castro MD, Perez-Juan PM, Garcia-Olmo J, Gomez-Nieto MA. Near infrared reflectance, spectroscopy and multivariate analysis in enology - Determination or screening of fifteen parameters in different types of wines. Anal Chim Acta. 2004;527:81-88.
41. Sauvage L, Frank D, Stearne J, Milikan MB. Trace metal studies of selected white wines and alternative approach. Anal Chim Acta. 2002;458:223-230.

42. Manley M, van Zyl A, Wolf EEH. The evaluation of the applicability of Fourier transform near-infrared (FT-NIR) sppectroscopy in the measurement of analytical parameters in must and wine. $S \mathrm{~A} J$ Oenol Viti. 2001;22:93-100.

43. Soriano PM, Pérez-Juan A, Vicario JM, González Pérez-Coello MS. Determination of anthocyanins in red wine using a newly developed method based on Fourier transform infrared spectroscopy. Food Chem. 2007;104:1295-1303.

44. Patz C-D, Blieke A, Ristow R, Dietrich H. Application of FT-MIR spectrometry in wine analysis. Anal Chim Acta. 2004;513:81-89.

45. Coimbra MA, Goncalves F, Barros AS, Delgadillo I. Fourier transform infrared spectroscopy and chemometric analysis of white wine polysaccharide extracts. J Agric Food Chem. 2002;50:3405-3411.

46. Lletí R, Meléndez E, Ortiz MC, Sarabia LA, Sánchez MS. Outliers in partial least squares regression: Application to calibration of wine grade with mean infrared data. Anal Chim Acta. 2005;544:60-70.

47. Nieuwoudt HH, Prior BA, Pretorius IS, Manley M, Bauer FF. Principal component analysis applied to Fourier transform infrared spectroscopy for the design of calibration sets for glycerol prediction models in wine and for the detection and classification of outlier samples. J Agric Food Chem. 2004;52:3726-3735.

48. Cozzolino D, Cynkar W, Janik L, Dambergs RG, Gishen M. Analysis of grape and wine by near infrared spectroscopy - a review. J Near Infrared Spectros. 2006;14:279-289.

49. Dixit V, Tewari JC, Cho B K, Irudayraj JMK. Identification and quantification of industrial grade glycerol adulteration in red wine with Fourier transform infrared spectroscopy using chemometrics and artificial neural networks. Appl Spectros. 2005;59:1553-1561.

50. Le Moigne M, Maury Ch, Bertrand D, Jourjon F. Sensory and instrumental characterisation of Cabernet Franc grapes according to ripening stages and growing location. Food Qual Pref. 2008;19:220-231.

51. Janik L, Cynkar WU, Cozzolino D, Dambergs RG, Gishen M. The prediction of total anthocyanin concentration in red-grape homogenates using near-infrared spectroscopy and artificial neural networks. Anal Chim Acta. 2007;594:107-118. 